\title{
Physicians' Understanding of Clinical Trial Data in Professional Prescription Drug Promotion
}

\author{
Caitlin K. Moynihan, BA, Panne A. Burke, MS, Sarah A. Evans, PhD, \\ Amie C. O'Donoghue, PhD, and Helen W. Sullivan, PhD, MPH
}

Background: Prescription drug marketing to physicians often includes clinical trial data. Prior research has shown that physicians may not have the necessary knowledge to understand all clinical trial results and they can be influenced by how these results are reported. This study focused on physicians' reactions to and evaluations of clinical trial data presented in professional prescription drug promotion.

Methods: We conducted 60-minute interviews with practicing physicians across the United States (50 primary care physicians and 22 endocrinologists). Physicians viewed prescription drug promotional materials that contained clinical trial data and answered follow-up questions.

Results: Physicians demonstrated low to moderate knowledge about clinical trial-related terms found in promotional prescription drug materials.

Discussion: Results from this qualitative analysis underscore a need to determine how clinical trial data in prescription drug promotional materials affect physicians' attitudes and decision making. ( $\mathrm{J}$ Am Board Fam Med 2018;31:645-649.)

Keywords: Endocrinologists, Marketing, Prescriptions, Primary Care Physicians

Prescription drug promotion to health care professionals is prevalent, with billions of dollars spent on it yearly. ${ }^{1}$ Exposure to this promotion has been correlated with physicians' increased prescribing frequency. ${ }^{2}$ Some studies have found an association with lower prescribing quality, but that is not a consistent finding. ${ }^{2}$ At the same time, several studies indicate that the way clinical trial results are reported can influence physicians, including their intent to prescribe. ${ }^{3-6}$ This may be a function of physicians' knowledge about clinical trial design or their experience with and skill in interpreting statistics. Surveys have found that physicians believe knowledge of biostatistics is important, but they

This article was externally peer reviewed.

Submitted 23 June 2017; revised 4 December 2017; accepted 10 December 2017.

From Fors Marsh Group, Arlington, VA (CKM, PAB, SAE); and US Food and Drug Administration, Silver Spring, MD (ACO, HWS).

Funding: This research was funded by the Office of Prescription Drug Promotion, U.S. Food and Drug Administration.

Conflict of interest: none declared.

Corresponding author: Helen W. Sullivan, PhD, MPH, Social Science Analyst, Food and Drug Administration, Silver Spring, MD 20993-0002 (E-mail: Helen.Sullivan@ fda.hhs.gov). have less knowledge than is needed to understand all clinical trial results. ${ }^{7-8}$ Rather, they tend to rely on informational framing in making prescribing decisions. For example, physicians tend to be more likely to prescribe a drug when results are framed as relative risk reduction instead of absolute risk reduction. ${ }^{6}$ Further, little is known about physicians' actual reactions to and evaluations of clinical trial data presented in professional prescription drug promotion. To this end, we conducted in-depth interviews with physicians to examine their understanding of clinical trial data, as presented in prescription drug promotional materials.

\section{Methods}

This study was approved by the Food and Drug Administration's Institutional Review Board. We conducted 60-minute interviews with 72 practicing physicians across the United States via telephone and computer. Participants were recruited through Doctor Directory, a former public-use search engine that connected researchers to health care providers who have opted in for potential studies. The final sample included primary care physicians $(\mathrm{n}=$ $50)$ and endocrinologists $(n=22)$ who wrote at 
Table 1. Final Participant Demographics

\begin{tabular}{|c|c|c|}
\hline Category & Classification & $\begin{array}{l}\text { Completed } \\
\text { Counts }\end{array}$ \\
\hline Total & & 72 \\
\hline \multirow[t]{2}{*}{ Physician type } & Primary care physician & 50 \\
\hline & Endocrinologist & 22 \\
\hline \multirow[t]{2}{*}{ Sex } & Male & 45 \\
\hline & Female & 27 \\
\hline \multirow[t]{4}{*}{ Race/ethnicity } & African American or black & 4 \\
\hline & Asian & 15 \\
\hline & Hispanic & 4 \\
\hline & White & 49 \\
\hline \multirow[t]{3}{*}{ Age } & 44 or Under & 29 \\
\hline & 45 to 64 & 37 \\
\hline & 65 or Older & 6 \\
\hline \multirow[t]{3}{*}{ Urbanicity } & $\begin{array}{l}\text { Large urban (eg, Washington, } \\
\text { DC) }\end{array}$ & 46 \\
\hline & Small urban (eg, St. Louis, MO) & 19 \\
\hline & $\begin{array}{l}\text { Suburban/rural (eg, Cortez, } \\
\text { CO) }\end{array}$ & 7 \\
\hline
\end{tabular}

least 50 prescriptions per week, allowing the sample to reach data saturation for reliable inferences. ${ }^{9} \mathrm{We}$ chose to include both primary care physicians and endocrinologists in the study to allow for potential comparisons based on the drug promotional materials chosen as study stimuli.

We used quotas to ensure geographic diversity and to reflect the American Medical Association's demographics (see Table 1 for final participant demographics). Remote interviews were conducted via telephone and web platform to facilitate stimuli sharing by a trained moderator skilled in leading semistructured discussions. Physicians were asked about their experience with and understanding of clinical trial data, as well as about any training they may have received in biostatistics and interpreting clinical trial data.

Following the initial background discussion, physicians viewed promotional materials for a prescription drug indicated for weight loss and another indicated for glycemic control in adults with diabetes mellitus and answered follow-up questions. The stimuli were promotional materials for actual prescription drugs (a slide deck and a sales aid). Due to time limitations, the stimuli were abbreviated. Content provided included the indication, common adverse reactions, contraindications, precautions and warnings, and clinical trial data. Exposure order was randomized, so that the participant only saw one stimulus set initially. With the first set, each participant was instructed to read the material as he or she normally would to evaluate the information. The moderator discussed the first set with each participant and asked specific questions. With the second set, the moderator focused on specific questions only. The materials contained clinical trial data concepts such as noninferiority and rerandomization. Four researchers coded interview transcripts into an organizational scheme for emergent themes by using NVivo (Ks $=0.83-$ 0.90). Following organizational coding, specific terminology responses were extracted for further coding on comprehension, and 2 coders categorized responses as accurate, inaccurate, or did not know $(K=0.70$; refer to Table 2$)$.

\section{Results}

In the background discussion, the majority of participants reported frequent exposure to clinical trial data via journals, publications, and promotional materials. In terms of training on clinical trial data, some participants reported receiving formal training during graduate school, medical school, residency, or fellowship. Others mentioned informal on-the-job training such as reading peer-reviewed journal articles. Most participants reported taking a statistics class that covered biostatistics involving clinical trial data during medical or graduate school. Although a few participants specified that their statistics classes covered the "basics" of interpreting clinical trial data, the majority of participants did not remember specific learnings.

Most participants reported being somewhat to fairly comfortable interpreting and applying clinical trial results; however, when participants were presented with the promotional stimuli, their responses indicated low to moderate knowledge of clinical trial data concepts. For 6 of the 9 terms within the stimuli examined, less than one-quarter of participants provided an accurate description (Table 2). Instead, a high percentage of participants said they did not know or provided responses that were not accurate (Table 2). Comparatively, greater understanding was associated with the terms randomized controlled trial, noninferiority, and rerandomization. More than half of the participants were unable to provide a response for the terms adjusted mean, per-protocol analysis, modified intent to treat, and last observation carried forward.

When asked what they typically do when encountering an unfamiliar concept or term while reading 


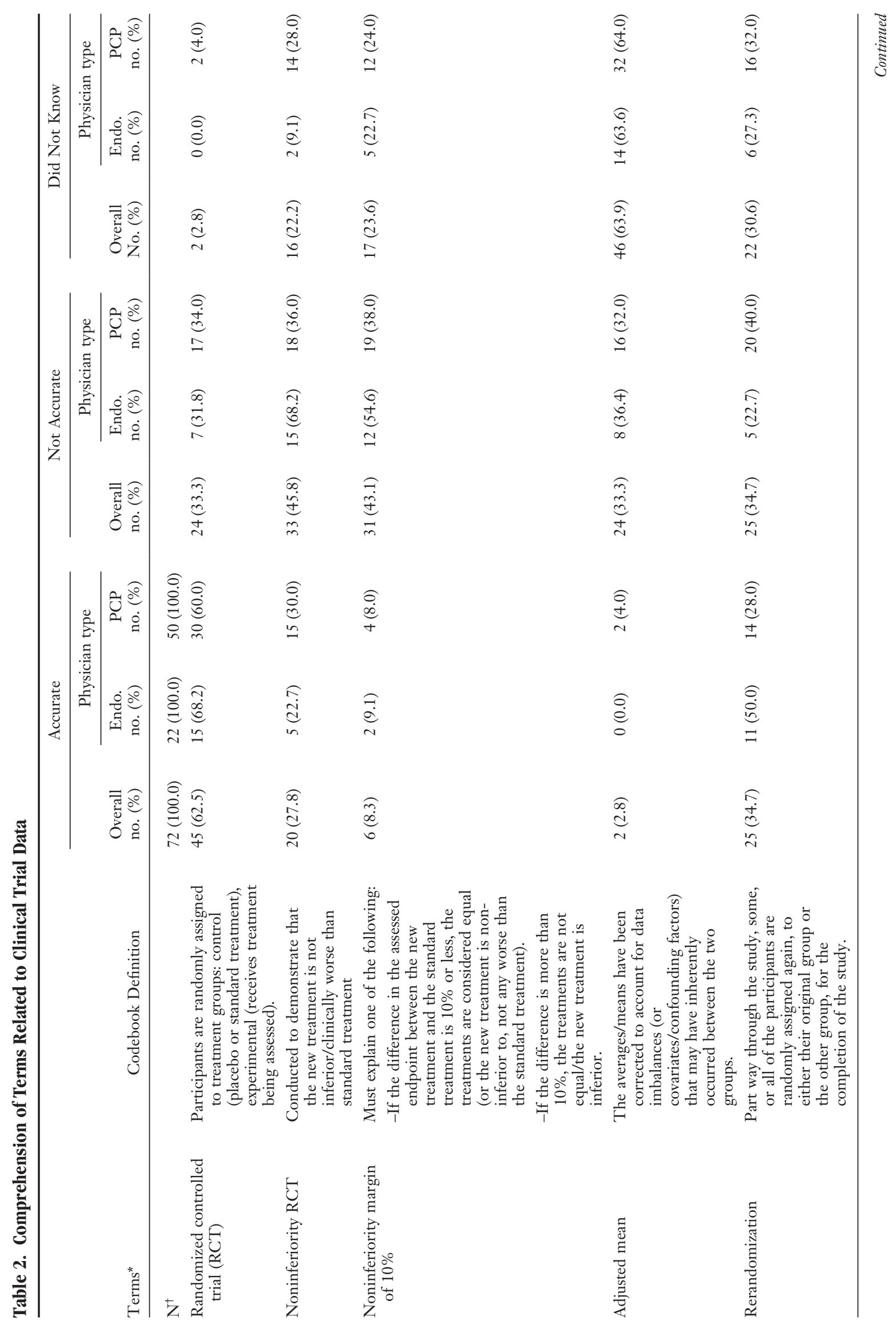




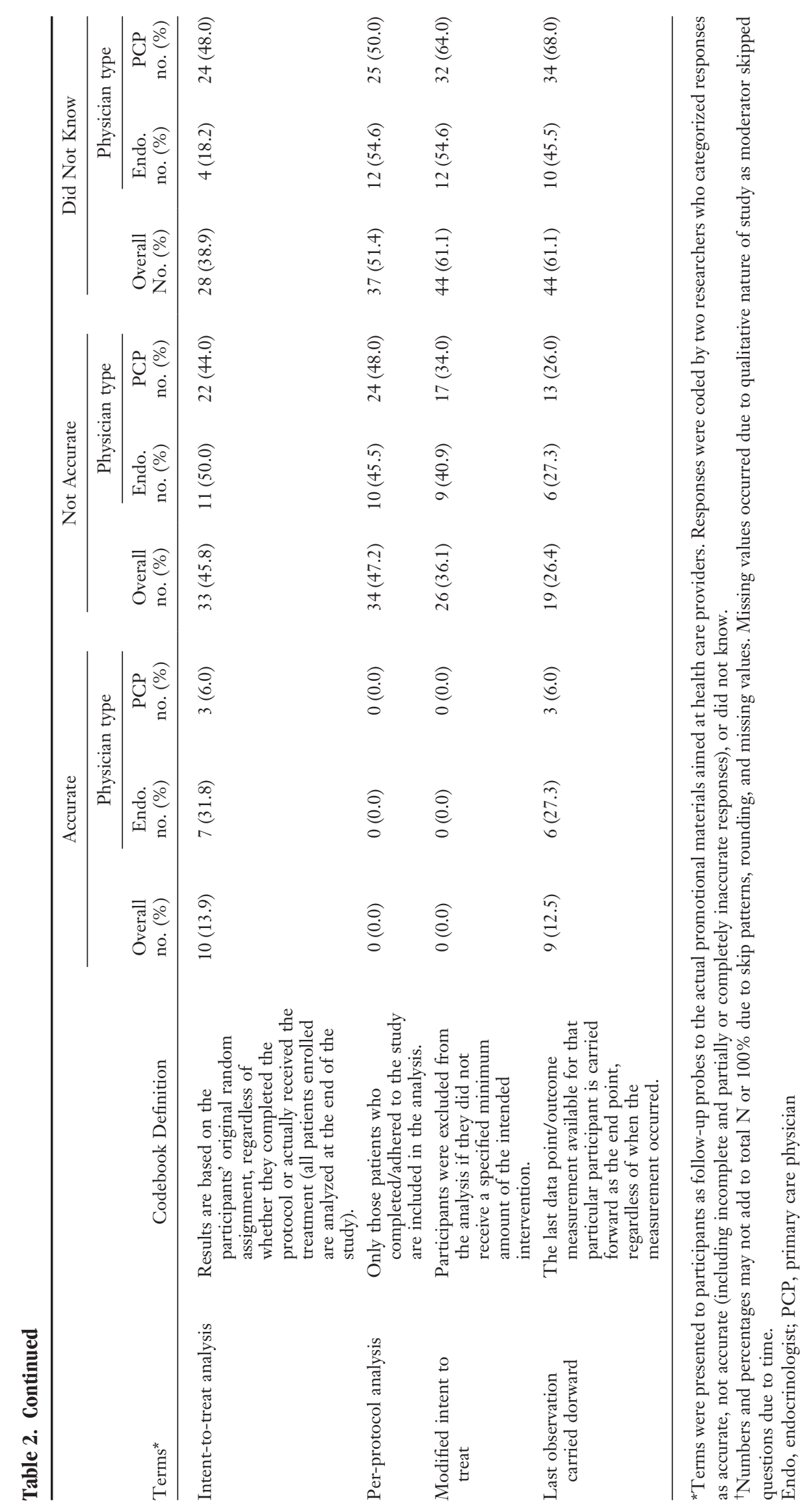


drug promotional materials, some participants reported that they often do not take further action, largely due to lack of time. A theme that emerged throughout the discussion was the reliability of the source when being presented with clinical trial data. Overall, participants expressed skepticism about the information in drug promotional materials, preferring to refer to online sources, published studies, regulatory authorities, and colleagues to find more objective and trustworthy information. Moreover, several participants indicated a level of distrust of statistics in stark contrast to substantial trust in colleagues' personal experiences, knowledge, and opinions when interpreting clinical trial data and evaluating unfamiliar pharmaceutical products. They further indicated consideration of a host of external factors when making prescription decisions, such as past experience, cost and insurance, patient-centered considerations (eg, pre-existing conditions, likely compliance), and safety.

\section{Discussion}

Overall, physicians demonstrated low to moderate knowledge when probed on specific clinical trial terms found in prescription drug promotional materials, despite prior exposure to clinical trial data from promotional materials and other publications, medical training inclusive of various statistics courses, and a general reported comfort in interpreting and applying clinical trial data.

Throughout the interviews, an important theme emerged: physicians tend to put less trust in promotional materials than other sources of information. Further, physicians tend to distrust statistics presented in promotional materials. These findings are curiously juxtaposed with the level of exposure physicians have to such materials and these materials' relationship with prescribing decisions documented in prior research. ${ }^{2}$

There are limitations due to the nature of the study. First, participants did not interact with the materials as they naturally would (eg, as part of a presentation), and they were primed, given that this was an interview, to expect follow-up questions. Thus, they may have examined the materials more thoroughly or looked at parts they would ordinarily ignore to respond to the moderator's probes. They were also asked to evaluate the information outside of the context of a specific patient. Second, our qualita- tive data are neither representative of nor generalizable to the primary care or endocrinologist population in the United States. Although the results are not statistically generalizable, we recruited a diverse sample of participants, and the total sample size was adequate to reach a point of data saturation. ${ }^{9}$ Finally, we could not test in this exploratory study whether low knowledge of clinical trial data terminology relates to the quality of treatment decisions or reliance on other sources of information.

This study adds to the literature that suggests physicians often have difficulty understanding clinical trial information. ${ }^{6}$ Instead of giving physicians a knowledge test, however, we asked them to interpret and react to clinical trial data presentations from actual promotional materials. Our findings underscore a need for further exploration of physicians' exposure to clinical trial data in prescription drug promotional materials and its relationship with attitudes and decision making.

To see this article online, please go to: bttp://jabfm.org/content/ 31/4/645.full.

\section{References}

1. Kornfield R, Donohue J, Berndt ER, Alexander GC. Promotion of prescription drugs to consumers and providers, 2001-2010. PLoS One 2013;8:e55504.

2. Spurling GK, Mansfield PR, Montgomery BD, et al. Information from pharmaceutical companies and the quality, quantity, and cost of physicians' prescribing: a systematic review. PLoS Med 2010;7:e1000352.

3. Marcatto F, Rolison JJ, Donatella F. Communicating clinical trial outcomes: effects of presentation method on physicians' evaluations of new treatments. Judgm Decis Mak 2013;8:29-33.

4. Bobbio M, Demichelis B, Giustetto G. Completeness of reporting trial results: effect on physicians' willingness to prescribe. Lancet 1994;343:1209-11.

5. Bucher $H$, Weinbacher $M$, Gyr K. Influence of method of reporting study results on decision of physicians to prescribe drugs to lower cholesterol concentration. BMJ 1994;309:761-4.

8. Kahwati L, Carmody D, Berkman N, Sullivan HW, Aikin KJ, DeFrank J. Prescribers' knowledge and skills for interpreting research results: a systematic review. J Contin Educ Health Prof 2017;37:129-36.

7. West CP, Ficalora RD. Clinician attitudes toward biostatistics. Mayo Clin Proc 2007;82:939-43.

8. Windish DM, Huot SJ, Green ML. Medicine residents' understanding of the biostatistics and results in the medical literature. JAMA 2007;298:1010-22.

9. Walker JL. The use of saturation in qualitative research. Can J Cardiovasc Nurs 2012;22:37-41. 